\title{
Hadron Masses in Medium and Neutron Star Properties
}

\author{
C. Y. Ryu ${ }^{a}$, C. H. Hyun ${ }^{a, b *}$, S. W. Hong ${ }^{a}$, B. K. Jennings ${ }^{c}$ \\ (a) Department of Physics and Institute of Basic Science, \\ Sungkyunkwan University, Suwon 440-746, Korea, \\ (b) School of Physics, Seoul Nat'l University, Seoul 151-742, Korea \\ (c) TRIUMF, 4004 Wesbrook Mall, Vancouver, \\ British Columbia, Canada V6T 2A3
}

(Dated: February 3, 2005)

\begin{abstract}
We investigate the properties of the neutron star with relativistic mean field models. We incorporate in the quantum hadrodynamics and in the quark-meson coupling models a possible reduction of meson masses in nuclear matter. The equation of state for neutron star matter is obtained and is employed in Oppenheimer-Volkov equation to extract the maximum mass of the stable neutron star. We find that the equation of state, the composition and the properties of the neutron stars are sensitive to the values of the meson masses in medium.
\end{abstract}

PACS numbers: 21.65.+f, 12.40.Yx, 26.60.+c, 12.39.Ki

Keywords: dense matter, in-medium meson mass, neutron star, quark model

*e-mail:hch@meson.skku.ac.kr 


\section{INTRODUCTION}

The state of matter at extremely hot or dense conditions is one of the most fundamental questions in physics. Terrestrial nuclei of heavy elements provide a glimpse at such extreme states, but there are far-more-extreme states of matter in our universe. A neutron star, which may be regarded as a huge nucleus, is one example of such an extreme state of matter. The state of matter can be characterized by the equation of state (EoS). From the microscopic point of view the EoS is determined by how the constituent particles of the matter interact with each other. Therefore the state of matter at extreme conditions can be understood when we have enough knowledge about the properties of the constituent particles and how they interact in such conditions.

In the early 90's, Brown and Rho proclaimed the scaling property of hadron masses in dense medium in terms of the scale invariance of the effective lagrangian [1]. They showed that the ratios of the in-medium masses of the nucleon, $\sigma-, \omega$ - and $\rho$-mesons to their masses in free space are approximately equal to each other at around the nuclear saturation density. Afterwards, changes in the hadron masses in medium were calculated in the framework of QCD-sum rule 2] and quark-meson-coupling (QMC) models [3]. In Refs. 2] and 3], masses of $\rho$ and $\omega$ mesons were calculated at the nuclear saturation density, and similar amount of mass reduction from the free mass, $m_{\rho, \omega}^{*} / m_{\rho, \omega} \simeq 0.8$ was predicted. Experimentally, microscopic properties of hadrons as well as the states of matter at hot or dense environment can be probed in relativistic heavy-ion collisions, and possible meson mass reductions in nuclear matter were reported through the dileptonic decay of $\rho$ and $\omega$ mesons in the CERES/NA45 [4] and KEK-PS E325 5] experiments. Also, recent astronomical observations of binary systems yield the mass of compact objects as $M_{\mathrm{J} 0751+1807}=(2.2 \pm$ $0.2) M_{\odot}$ [6] and $M_{4 \mathrm{U} 1700-37}=(2.44 \pm 0.27) M_{\odot}$ [7], of which the former is believed to be a neutron star. Such a large mass of the neutron star requires a substantially stiff EoS.

Motivated by these theoretical and experimental studies, in this work we take into account the reduction of meson masses in the nuclear models such as quantum hadrodynamics (QHD) and QMC, and apply these models to the neutron star matter. The nuclear models are calibrated to the bulk properties of nuclear matter at the saturation density, such as saturation density $\rho_{0}\left(=0.17 \mathrm{fm}^{3}\right)$, binding energy $E_{B}(=16.0 \mathrm{MeV})$, symmetry energy $a_{\text {sym }}$ $(=32.5 \mathrm{MeV})$ and compression modulus $K(200 \sim 300 \mathrm{MeV})$. We take into account the 
effect of meson mass changes in the following ways. First, we use the meson masses in free space for the nuclear models considered here as is usually done in most works. Secondly, we adopt the Brown-Rho (BR) scaling law [1],

$$
\frac{m_{N}^{*}}{m_{N}} \simeq \frac{m_{\sigma}^{*}}{m_{\sigma}} \simeq \frac{m_{\omega}^{*}}{m_{\omega}} \simeq \frac{m_{\rho}^{*}}{m_{\rho}}
$$

and assume a density-dependent scaling function for the meson masses [8]. The nuclear models that employ the scaling function are denoted by the abbreviation "S". Thirdly, in using the QMC type models we may treat the heavy mesons ( $\rho$ and $\omega$ ) as meson bags (denoted by "MB") composed of a quark and an anti-quark [9]. In such cases, heavy mesons are treated in consistent with the description of the nucleon in the framework of the QMC. After calibrating different nuclear models for nuclear matter we apply them to the neutron star matter and see how the reduction of meson masses in matter may influence the EoS of the neutron star matter and the properties of the neutron star.

In Sec. 2, we briefly describe the nuclear models considered in this work and fix the parameters of the models and show the resultant properties of the nuclear matter at the saturation density. In Sec. 3, we apply the various nuclear models fixed in Sec. 2 to the neutron star matter and discuss the results and implications for the properties of the neutron star. A summary is given in Sec. 4.

\section{MODELS FOR NUCLEAR MATTER}

In this work we will consider 5 different models. We refer to them as QHD, QHD-S; MQMC, MQMC-S and MQMC-MB. All the models have three coupling constants $g_{\sigma N}$, $g_{\omega N}$, and $g_{\rho N}$ (or $g_{\sigma}^{q}, g_{\omega}^{q}$, and $g_{\rho}^{q}$ ) for the interaction of nucleons (or quarks) with $\sigma-, \omega$ - and $\rho$-mesons, respectively. $\sigma$ - and $\omega$-coupling constants are fitted to reproduce the given values of $\rho_{0}$ and $E_{B}$, and the coupling to $\rho$-meson is determined by $a_{\text {sym }}$. Details of each model are explained in the following subsections.

\section{A. QHD}

The lagrangian for QHD in the mean field approximation reads

$$
L_{\mathrm{QHD}}^{\mathrm{MF}}=\bar{\psi}_{N}\left[i \gamma^{\mu} \partial_{\mu}-\left(m_{N}-g_{\sigma N} \bar{\sigma}\right)-g_{\omega N} \gamma^{0} \bar{\omega}_{0}-\frac{1}{2} g_{\rho N} \gamma^{0} \bar{b}_{03} \tau_{3}\right] \psi_{N}
$$




$$
\begin{aligned}
& -\frac{1}{2} m_{\sigma}^{2} \bar{\sigma}^{2}-\frac{1}{3} m_{N} b\left(g_{\sigma N} \bar{\sigma}\right)^{3}-\frac{1}{4} c\left(g_{\sigma N} \bar{\sigma}\right)^{4} \\
& +\frac{1}{2} m_{\omega}^{2} \bar{\omega}_{0}^{2}+\frac{1}{2} m_{\rho}^{2} \bar{b}_{03}^{2} .
\end{aligned}
$$

The masses $m_{N}, m_{\sigma}, m_{\omega}$ and $m_{\rho}$ refer to the values in free space, 939, 550, 783 and 770 $\mathrm{MeV}$, respectively. The effective mass of the nucleon is defined as

$$
m_{N}^{*}(\mathrm{QHD}) \equiv m_{N}-g_{\sigma N} \bar{\sigma}
$$

The meson fields are determined from the equations of motion in the mean field approximation ;

$$
\begin{aligned}
\bar{\sigma} & =\frac{g_{\sigma N}}{m_{\sigma}^{2}} \rho_{s}-\frac{m_{N}}{m_{\sigma}^{2}} b g_{\sigma N}^{3} \bar{\sigma}^{2}-\frac{1}{m_{\sigma}^{2}} c g_{\sigma N}^{4} \bar{\sigma}^{3}, \\
\bar{\omega}_{0} & =\frac{g_{\omega N}}{m_{\omega}^{2}} \frac{1}{3 \pi^{2}} \sum_{N=n, p} k_{N}^{3}=\frac{g_{\omega N}}{m_{\omega}^{2}} \rho, \\
\bar{b}_{03} & =\frac{g_{\rho N}}{m_{\rho}^{2}} \frac{1}{3 \pi^{2}} \sum_{N=n, p} I_{N 3} k_{N}^{3}=\frac{1}{2} \frac{g_{\rho N}}{m_{\rho}^{2}}\left(\rho_{p}-\rho_{n}\right),
\end{aligned}
$$

where $I_{N 3}$ is $1 / 2(-1 / 2)$ for the proton (neutron).

$$
\rho_{s}=\frac{1}{\pi^{2}} \sum_{N=n, p} \int_{0}^{k_{N}} \frac{m_{N}^{*}}{\sqrt{k^{2}+m_{N}^{* 2}}} k^{2} d k
$$

is the scalar density and $k_{N}$ is the Fermi momentum of the nucleon at a given density. Cubic and quartic self-interaction terms of the $\sigma$-meson are included in Eq. (2) to produce reasonable $K$ and $m_{N}^{*}$ values at the saturation density. The coupling constants fixed to produce the saturation properties and the resulting $m_{N}^{*}$ and $K$ are listed in Tab. I.

\section{B. QHD-S}

We now consider the meson mass changes in matter and adopt the model proposed in Ref. 8] where the BR-scaling law is incorporated in the original QHD. The model lagrangian reads

$$
\begin{aligned}
L_{\mathrm{QHD}-\mathrm{S}}^{\mathrm{MF}}= & \bar{\psi}_{N}\left[i \gamma^{\mu} \partial_{\mu}-\left(M_{N}^{*}-g_{\sigma N} \bar{\sigma}\right)-g_{\omega N}^{*} \gamma^{0} \bar{\omega}_{0}-\frac{1}{2} g_{\rho N} \gamma^{0} \bar{b}_{03} \tau_{3}\right] \psi_{N} \\
& -\frac{1}{2} m_{\sigma}^{* 2} \bar{\sigma}^{2}+\frac{1}{2} m_{\omega}^{* 2} \bar{\omega}_{0}^{2}+\frac{1}{2} m_{\rho}^{* 2} \bar{b}_{03}^{2} .
\end{aligned}
$$

The BR-scaling law is parametrized as [8]

$$
\frac{M_{N}^{*}}{m_{N}}=\frac{m_{\sigma}^{*}}{m_{\sigma}}=\frac{m_{\omega}^{*}}{m_{\omega}}=\frac{m_{\rho}^{*}}{m_{\rho}}=\left(1+y \frac{\rho}{\rho_{0}}\right)^{-1} .
$$




\begin{tabular}{|c|c|c|c|c|c|c|c||c|c|c|}
\hline Model & $g_{\sigma N}$ & $g_{\omega N}$ & $g_{\rho N}$ & $b \times 100$ & $c \times 100$ & $y$ & $z$ & $m_{N}^{*} / m_{N}$ & $m_{M}^{*} / m_{M}$ & $K$ \\
\hline \hline QHD & 8.11 & 8.36 & 7.85 & 0.3478 & 1.328 & $\cdot$ & $\cdot$ & 0.773 & 1.0 & 310.8 \\
\hline QHD-S & 5.30 & 15.30 & 7.52 & $\cdot$ & $\cdot$ & 0.28 & 0.31 & 0.667 & 0.781 & 264.5 \\
\hline
\end{tabular}

TABLE I: Parameters and the saturation properties for the QHD and QHD-S models. The subscript $M$ for $m_{M}^{*} / m_{M}$ refers to $\sigma, \omega$ and $\rho$ mesons.

The effective mass of the nucleon is defined as

$$
m_{N}^{*}(\mathrm{QHD}-\mathrm{S})=M_{N}^{*}-g_{\sigma N} \bar{\sigma}
$$

(Note the use of $M_{N}^{*}$ in place of $m_{N}$ of Eq. (3).) Coupling constants may change with nuclear matter density. Such density dependences of coupling constants as well as masses have been considered in previous [10, 11, 12] and very recent [13, 14] works within the framework of relativistic mean field theory. In Ref. [8], $g_{\omega N}$ is assumed to vary with density to satisfy $g_{\omega N}^{*} / g_{\omega N} \simeq m_{\omega}^{*} / m_{\omega}$ at around the saturation density. $g_{\omega N}^{*}$ scaling is expressed as [8]

$$
\frac{g_{\omega N}^{*}}{g_{\omega N}}=\left(1+z \frac{\rho}{\rho_{0}}\right)^{-1} .
$$

The parameters for the QHD-S model and the resulting properties at the saturation are summarized in Tab. II. One can see that both QHD and QHD-S models give $K$ values in the acceptable range. $m_{M}^{*} / m_{M}$ turns out to be 0.781 , which is close to the values obtained by previous studies [2, 3]. $m_{N}^{*} / m_{N}$ from QHD-S is smaller than that from QHD because of the scaling in Eq. (9).

\section{MQMC}

In the QMC model [15], interactions between the nucleons (or bags) are mediated by the exchange of mesons which couple with the quarks in the nucleon bags. The mean field lagrangian for non-overlapping spherical bags in dense matter may be written as

$$
\begin{aligned}
L_{\mathrm{QMC}}^{\mathrm{MF}}= & \bar{\psi}_{q}\left[i \gamma^{\mu} \partial_{\mu}-\left(m_{q}^{0}-g_{\sigma}^{q} \bar{\sigma}\right)-g_{\omega}^{q} \gamma^{0} \bar{\omega}_{0}-\frac{1}{2} g_{\rho}^{q} \gamma^{0} \bar{b}_{03} \tau_{3}-B\right] \times \theta_{V}(R-r) \psi_{q} \\
& -\frac{1}{2} m_{\sigma}^{2} \bar{\sigma}^{2}+\frac{1}{2} m_{\omega}^{2} \bar{\omega}_{0}^{2}+\frac{1}{2} m_{\rho}^{2} \bar{b}_{03}^{2}
\end{aligned}
$$

where $g_{M}^{q}(q=u, d ; M=\sigma, \omega, \rho)$ is the coupling constant for the quark-meson interaction. $B$ is the bag constant and $R$ is the bag radius within which quarks are confined. The effective 
mass of the nucleon in the QMC is given by

$$
\begin{aligned}
m_{N}^{*}(\mathrm{QMC}) & =\sqrt{\left(E_{b a g}^{N}\right)^{2}-\sum_{q} \frac{x_{q}^{2}}{R^{2}}}, \\
E_{b a g}^{N} & =\sum_{q} \frac{\Omega_{q}}{R}-\frac{Z_{N}}{R}+\frac{4}{3} \pi R^{3} B, \\
\Omega_{q} & =\sqrt{x_{q}^{2}+R^{2} m_{q}^{* 2}}, \quad\left(m_{q}^{*}=m_{q}^{0}-g_{\sigma}^{q} \bar{\sigma}\right) .
\end{aligned}
$$

$x_{q}$ is the eigen energy of the quarks in the bag determined by the boundary conditions at $r=R . Z_{N}$ is a phenomenological constant that incorporates the effects not explicitly taken into account, including zero-point motion.

The scalar and the vector potentials obtained from the QMC model were much smaller than those from the QHD, which would result in too weak a spin-orbit potential to explain the spin-orbit splittings in finite nuclei and spin observables in nucleon-nucleus scattering. To circumvent these shortcomings, density dependent bag constants were introduced [16]. The QMC model with a density dependent bag constant is called the modified QMC (MQMC) model. We employ the direct coupling model of Ref. 16] where the bag constant reads

$$
B=B_{0}\left(1-g_{\sigma}^{B} \frac{4}{\delta} \frac{\bar{\sigma}}{m_{N}}\right)^{\delta}
$$

$\bar{\sigma}$ value is determined from the self-consistency condition (SCC)

$$
\bar{\sigma}=3 \frac{g_{\sigma}^{q}}{m_{\sigma}^{2}} \rho_{s}\left[C_{N}(\bar{\sigma})+\frac{g_{\sigma}^{B}}{g_{\sigma}^{q}} \frac{E_{b a g}^{N}}{m_{N}^{*}} \frac{16 \pi}{9} R^{3} \frac{B}{m_{N}}\left(1-\frac{4}{\delta} \frac{g_{\sigma}^{B} \bar{\sigma}}{m_{N}}\right)^{-1}\right]
$$

with

$$
C_{N}(\bar{\sigma})=\frac{E_{b a g}^{N}}{m_{N}^{*}}\left[\left(1-\frac{\Omega_{q}}{E_{b a g}^{N} R}\right) S(\bar{\sigma})+\frac{m_{q}^{*}}{E_{b a g}^{N}}\right]
$$

and

$$
S(\bar{\sigma})=\frac{\Omega_{q} / 2+R m_{q}^{*}\left(\Omega_{q}-1\right)}{\Omega_{q}\left(\Omega_{q}-1\right)+R m_{q}^{*} / 2} .
$$

$B_{0}$ and $Z_{N}$ are fitted to reproduce the free nucleon mass with the stability condition

$$
\left.\frac{\partial m_{N}^{*}}{\partial R}\right|_{R=R_{0}}=0
$$

where $m_{N}^{*}$ is evaluated by Eq. (13). Choosing $R_{0}=0.6 \mathrm{fm}$, we obtain $B_{0}^{1 / 4}=188.1 \mathrm{MeV}$ and $Z_{N}=2.030 . g_{\sigma}^{B}$ and $\delta, g_{\sigma}^{q}$ and $g_{\omega}^{q}$ are adjusted to produce saturation properties and reasonable values of $K$ and $m_{N}^{*} \cdot g_{\rho}^{q}$ is fitted for the symmetry energy to be reproduced. 


\section{MQMC-S}

As a simple way to incorporate the reduction of meson mass in medium, we assume

$$
\frac{m_{\sigma}^{*}}{m_{\sigma}}=\frac{m_{\omega}^{*}}{m_{\omega}}=\frac{m_{\rho}^{*}}{m_{\rho}}=\left(1+y \frac{\rho}{\rho_{0}}\right)^{-1} .
$$

The scaling parameter $y$ is determined to satisfy Eq. (11) at around the saturation density with $m_{N}^{*}$ given by Eq. (13). The lagrangian for this model can be easily obtained by replacing the meson masses in Eq. (12) with the medium-modified values in Eq. (21). Since the SCC is obtained from the minimization of the energy density with respect to the variation of $\bar{\sigma}$, the SCC of the MQMC-S is of the same form as that of MQMC, but with $m_{\sigma}$ replaced by $m_{\sigma}^{*}$ given by Eq. (21). The model parameters fixed to produce saturation properties are listed in Tab. III

\section{E. MQMC-MB}

In the framework of MQMC, we may treat vector mesons as MIT bags composed of a quark and an anti-quark. We refer to such a model as MQMC-MB. The parameters $B_{0}$, $R_{0}$ and $Z_{M}(M=\rho, \omega)$ for the meson bags can differ depending on the mesons, but for simplicity we fix $B_{0}$ and $R_{0}$ as those values for the nucleon and treat only $Z_{M}$ as a parameter for each meson [9]. The effective mass of each meson can be written as

$$
\begin{aligned}
& m_{M}^{*}=\sqrt{\left(E_{b a g}^{M}\right)^{2}-2 \frac{x_{q}^{2}}{R^{2}}}, \\
& E_{b a g}^{M}=2 \frac{\Omega_{q}}{R}-\frac{Z_{M}}{R}+\frac{4}{3} \pi R^{3} B .
\end{aligned}
$$

$Z_{M}$ is fixed so that $m_{M}^{*}$ defined as in Eq. (22) recovers the mass of the $\omega$ - and $\rho$-mesons in free space. $Z_{\omega}=0.7904$ and $Z_{\rho}=0.8154$ are obtained. The lagrangian for the model can be written by replacing the meson masses in Eq. (12) with $m_{M}^{*}$ in Eq. (22). In the MQMC-MB meson masses are functions of $\bar{\sigma}$, which modifies the SCC as

$$
\begin{aligned}
\bar{\sigma} & =3 \frac{g_{\sigma}^{q}}{m_{\sigma}^{2}} \rho_{s}\left[C_{N}(\bar{\sigma})+\frac{g_{\sigma}^{B}}{g_{\sigma}^{q}} \frac{E_{b a g}^{N}}{m_{N}^{*}} \frac{16 \pi}{9} R^{3} \frac{B}{m_{N}}\left(1-\frac{4}{\delta} \frac{g_{\sigma}^{B} \bar{\sigma}}{m_{N}}\right)^{-1}\right] \\
& -18 \frac{g_{\sigma}^{q}}{m_{\sigma}^{2}} \frac{g_{\omega}^{q 2}}{m_{\omega}^{* 3}} \rho^{2}\left[C_{\omega}(\bar{\sigma})+\frac{g_{\sigma}^{B}}{g_{\sigma}^{q}} \frac{E_{b a g}^{\omega}}{m_{\omega}^{*}} \frac{8 \pi}{3} R^{3} \frac{B}{m_{N}}\left(1-\frac{4}{\delta} \frac{g_{\sigma}^{B} \bar{\sigma}}{m_{N}}\right)^{-1}\right] \\
& -2 \frac{g_{\sigma}^{q}}{m_{\sigma}^{2}} \frac{g_{\rho}^{q 2}}{m_{\rho}^{* 3}}\left(\sum_{N=n, p} I_{N 3} \rho_{N}\right)^{2}\left[C_{\rho}(\bar{\sigma})+\frac{g_{\sigma}^{B}}{g_{\sigma}^{q}} \frac{E_{b a g}^{\rho}}{m_{\rho}^{*}} \frac{8 \pi}{3} R^{3} \frac{B}{m_{N}}\left(1-\frac{4}{\delta} \frac{g_{\sigma}^{B} \bar{\sigma}}{m_{N}}\right)^{-1}\right]
\end{aligned}
$$




\begin{tabular}{|c|c|c|c|c|c||c|c|c|}
\hline Model & $g_{\sigma}^{q}$ & $g_{\omega}^{q}$ & $g_{\sigma}^{B}$ & $g_{\rho}^{q}$ & $y$ & $m_{N}^{*} / m_{N}$ & $m_{M}^{*} / m_{M}$ & $K$ \\
\hline MQMC & 1.0 & 2.71 & 6.81 & 7.89 &. & 0.783 & 1.0 & 285.5 \\
\hline MQMC-S & 1.0 & 2.31 & 5.51 & 6.09 & 0.28 & 0.758 & 0.781 & 591.5 \\
\hline MQMC-MB & 1.0 & 1.77 & 5.44 & 8.15 &. & 0.852 & 0.861 & 324.1 \\
\hline
\end{tabular}

TABLE II: The parameters and the saturation properties from MQMC-type models. The subscript $M$ denotes $\sigma, \omega$ and $\rho$ mesons for MQMC-S. For MQMC-MB, $M$ refers to $\omega$ and $\rho$ mesons. $B_{0}^{1 / 4}=188.1 \mathrm{MeV}$ and $Z_{N}=2.030$ are used for all three cases.

where

$$
C_{M}(\bar{\sigma}) \equiv \frac{E_{b a g}^{M}}{m_{M}^{*}}\left[\left(1-\frac{\Omega_{q}}{E_{b a g}^{M} R}\right) S(\bar{\sigma})+\frac{m_{q}^{*}}{E_{b a g}^{M}}\right] .
$$

The parameters that can produce the saturation at $\rho_{0}$ are listed in Tab. III MQMC and MQMC-MB give us acceptable results for $K$ and $m_{N}^{*}$, but the $K$ value for MQMC-S is as large as twice of usually accepted ranges of values. To get a value of $K$ within a reasonable range, we have tried many numerical searches over a wide range of parameter space, but no solution has been found so far.

\section{APPLICATION TO THE NEUTRON STAR}

We now apply the five nuclear models described in Sect. II to the neutron star matter. Two basic assumptions of the neutron star matter are the charge neutrality and the $\beta$-equilibrium. If we assume that only the nucleons and light leptons exist in the neutron star, charge neutrality is expressed as

$$
\rho_{p}=\sum_{l=e, \mu} \rho_{l}
$$

where $\rho_{i}$ is the number density of particle $i(=p, e, \mu)$. Under $\beta$-equilibrium, the processes

$$
n \rightarrow p+e^{-}+\bar{\nu}_{e} \text { and } p+e^{-} \rightarrow n+\nu_{e}
$$

occur at the same rate. The condition can be satisfied when the chemical potentials before and after the decay are the same. The chemical potential of each particle reads

$$
\mu_{n}=\sqrt{k_{n}^{2}+m_{N}^{* 2}}+g_{\omega N} \bar{\omega}_{0}-\frac{1}{2} g_{\rho N} \bar{b}_{03}
$$




$$
\begin{aligned}
\mu_{p} & =\sqrt{k_{p}^{2}+m_{N}^{* 2}}+g_{\omega N} \bar{\omega}_{0}+\frac{1}{2} g_{\rho N} \bar{b}_{03}, \\
\mu_{l} & =\sqrt{k_{l}^{2}+m_{l}^{2}}
\end{aligned}
$$

where $k_{l}$ is the Fermi momentum of the lepton $l(=e, \mu)$. The chemical equilibrium condition is expressed as

$$
\mu_{n}=\mu_{p}+\sum_{l} \mu_{l}
$$

\section{A. $\mathbf{E o S}$}

The EoS tells us the pressure $(P)$ as a function of energy density $(\varepsilon)$, which are the diagonal elements of the energy-momentum tensor. The energy density and the pressure in the QHD model read

$$
\begin{aligned}
\varepsilon & =\frac{1}{2} m_{\sigma}^{2} \bar{\sigma}^{2}+\frac{1}{3} m_{N} b\left(g_{\sigma N} \bar{\sigma}\right)^{3}+\frac{1}{4} c\left(g_{\sigma N} \bar{\sigma}\right)^{4}+\frac{1}{2} m_{\omega}^{2} \bar{\omega}_{0}^{2}+\frac{1}{2} m_{\rho}^{2} \bar{b}_{03}^{2} \\
& +\frac{1}{\pi^{2}} \sum_{N=n, p} \int_{0}^{k_{N}} \sqrt{k^{2}+m_{N}^{* 2}} k^{2} d k+\frac{1}{\pi^{2}} \sum_{l} \int_{0}^{k_{l}} \sqrt{k^{2}+m_{l}^{2}} k^{2} d k, \\
P & =-\frac{1}{2} m_{\sigma}^{2} \bar{\sigma}^{2}-\frac{1}{3} m_{N} b\left(g_{\sigma N} \bar{\sigma}\right)^{3}-\frac{1}{4} c\left(g_{\sigma N} \bar{\sigma}\right)^{4}+\frac{1}{2} m_{\omega}^{2} \bar{\omega}_{0}^{2}+\frac{1}{2} m_{\rho}^{2} \bar{b}_{03}^{2} \\
& +\frac{1}{3 \pi^{2}} \sum_{N=n, p} \int_{0}^{k_{N}} \frac{k^{4}}{\sqrt{k^{2}+m_{N}^{* 2}}} d k+\frac{1}{3 \pi^{2}} \sum_{l} \int_{0}^{k_{l}} \frac{k^{4}}{\sqrt{k^{2}+m_{l}^{2}}} d k .
\end{aligned}
$$

The energy density and the pressure from the QHD-S model can be obtained from Eq. (32) and Eq. (33), respectively, by removing the cubic and quartic self-interaction terms of the $\sigma$ meson and replacing the free meson masses with the scaled ones given in Eq. (21). However the pressure from the QHD-S model has additional terms that stem from the density dependent meson masses and coupling constants. Thermodynamic consistency of these terms is discussed in Ref. [17], and the explicit form is given as

$$
\begin{aligned}
P & =\frac{1}{3 \pi^{2}} \sum_{N=n, p} \int_{0}^{k_{N}} \frac{k^{4}}{\sqrt{k^{2}+m_{N}^{* 2}}} d k+\frac{1}{3 \pi^{2}} \sum_{l} \int_{0}^{k_{l}} \frac{k^{4}}{\sqrt{k^{2}+m_{l}^{2}}} d k \\
& -\frac{1}{2} m_{\sigma}^{* 2} \bar{\sigma}^{2}+\frac{1}{2} m_{\omega}^{* 2} \bar{\omega}_{0}^{2}+\frac{1}{2} m_{\rho}^{* 2} \bar{b}_{03}^{2}-m_{\sigma}^{* 2} \bar{\sigma}^{2} \frac{y}{1+y \frac{\rho}{\rho_{0}}} \frac{\rho}{\rho_{0}}-m_{\sigma}^{* 2} \bar{\sigma} \frac{m_{N}}{g_{\sigma N}} \frac{y}{\left(1+y \frac{\rho}{\rho_{0}}\right)^{2}} \frac{\rho}{\rho_{0}} \\
& +m_{\omega}^{* 2} \bar{\omega}_{0}^{2}\left(\frac{y}{1+y \frac{\rho}{\rho_{0}}}-\frac{z}{1+z \frac{\rho}{\rho_{0}}}\right) \frac{\rho}{\rho_{0}}+m_{\rho}^{* 2} \bar{b}_{03}^{2} \frac{y}{1+y \frac{\rho}{\rho_{0}}} \frac{\rho}{\rho_{0}} .
\end{aligned}
$$



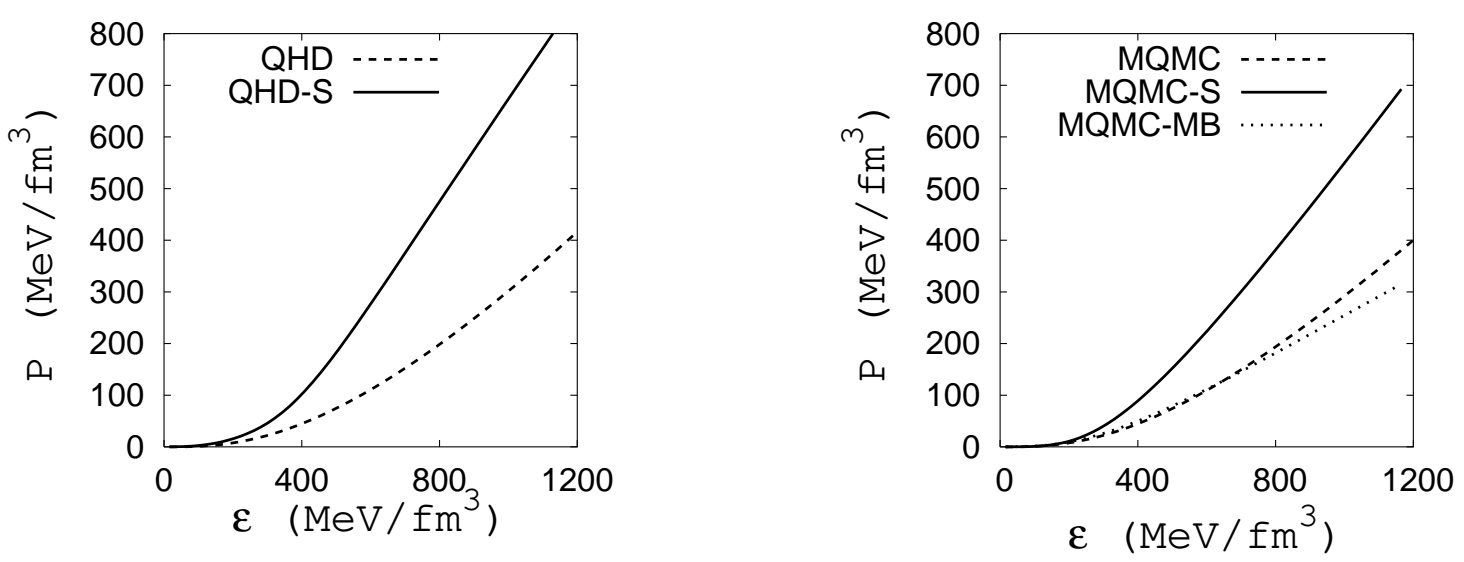

FIG. 1: EoS from five different models.

The energy density and the pressure of the MQMC-type models can be obtained by using $g_{\sigma N}=3 g_{\sigma}^{q}, g_{\omega N}=3 g_{\omega}^{q}$ and $g_{\rho N}=g_{\rho}^{q}$ together with proper replacement of in-medium meson masses in Eqs. (32) and (34). The resulting EoS curves for each model are shown in Fig. (1) If the pressure exerted by nuclear repulsion is strong, the matter becomes more incompressible, which corresponds to a large compression modulus and a stiff EoS. The EoS's from the QHD, MQMC and MQMC-MB models whose $K$ 's are close to each other $(310.8,285.5$ and 324.1 $\mathrm{MeV}$, respectively) show similar behaviors at the energy densities considered here.

The EoS from the scaling models (QHD-S and MQMC-S) turns out to be stiffer than the EoS from other models. This behavior can be understood by observing that the repulsion from the $\omega$-meson is augmented at high densities (see Eq. (15) with $m_{\omega}$ replaced by $m_{\omega}^{*}$ ) while the attraction caused by the $\sigma$-meson is not strong enough to cancel the repulsion.

To illustrate this argument clearly, we plot in Fig. 2 the magnitude of the contributions to the pressure $P$ from $N, \sigma, \omega$ and $\rho$. Each contribution $P_{i}(i=N, \sigma, \omega, \rho)$ is defined as

$$
\begin{aligned}
P_{N} & =\frac{1}{3 \pi^{2}} \sum_{N=n, p} \int_{0}^{k_{N}} \frac{k^{4}}{\sqrt{k^{2}+m_{N}^{* 2}}} d k, \\
P_{\sigma} & =\frac{1}{2} m_{\sigma}^{* 2} \bar{\sigma}^{2}, \quad P_{\omega}=\frac{1}{2} m_{\omega}^{* 2} \bar{\omega}_{0}^{2}, \quad P_{\rho}=\frac{1}{2} m_{\rho}^{* 2} \bar{b}_{30}^{2} .
\end{aligned}
$$

The total pressure is approximately

$$
P \simeq-P_{\sigma}+P_{\omega}+P_{\rho}+P_{N}
$$

The remaining terms such as the cubic and quartic self-interaction terms in QHD, extra terms for thermodynamic consistency and lepton contributions can be neglected since they 

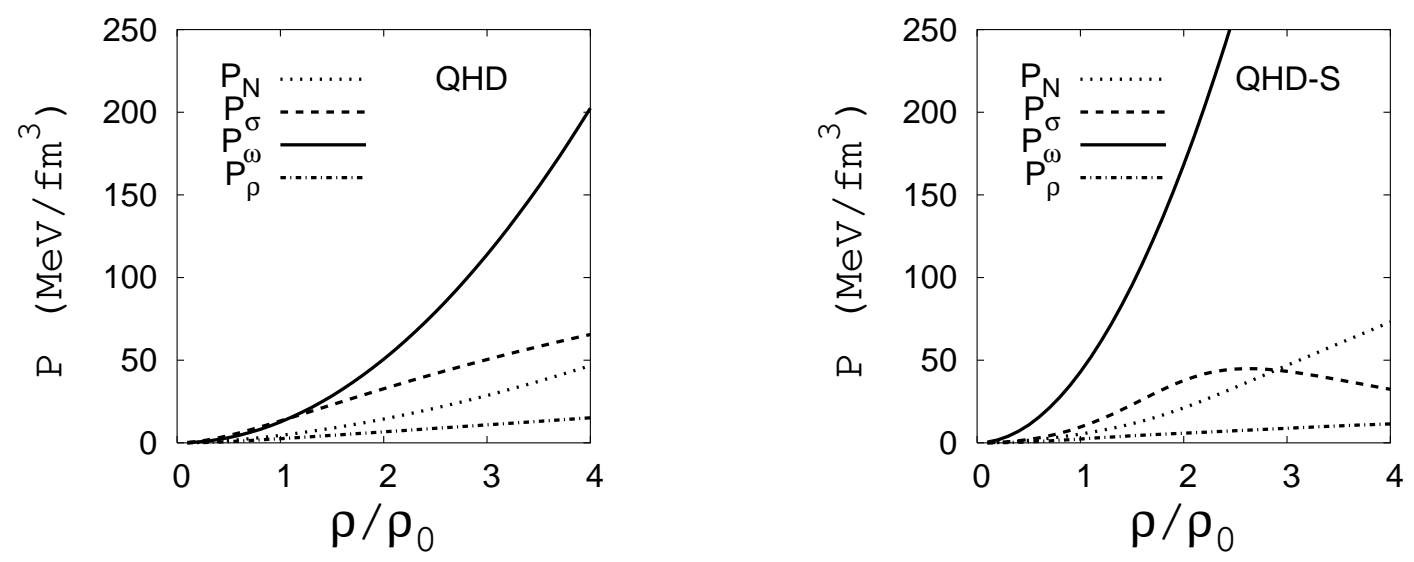

FIG. 2: Comparison of the magnitude of $\sigma, \omega, \rho$ and the nucleon contributions to the pressure for the QHD (left) and the QHD-S (right). Definitions of $P_{i}$ 's are given in the text.

do not determine the overall behavior of EoS. In the case of the QHD (see the left panel of Fig. 2), $P_{\sigma}$ is non-negligible compared to $P_{\omega}$ in the density region considered here. Since $\sigma$-meson contributes to the pressure negatively, $P_{\sigma}$ reduces the total pressure substantially, which in turn leads to a sizable softening of the EoS. On the other hand, $P_{\omega}$ from QHD-S is about 3 times larger than that of QHD, but $P_{\sigma}$ from QHD-S is more or less similar to that from the QHD. Thus the softening of the EoS due to $P_{\sigma}$ in QHD-S is relatively weak as the density becomes high, and consequently the EoS from QHD-S is stiffer than that from QHD.

Here, we need to remark that in Fig. 1 the EoS curves for MQMC-S and MQMC-MB terminate at a certain $\varepsilon$. It is due to a breakdown of the SCC at a certain density. In Appendix, we show the details of why the SCC does not have a solution at some density.

\section{B. Composition}

The composition of the neutron star matter is represented by the number of particles divided by the total baryon number. The number of each species of particles is determined by the charge neutrality and the chemical equilibrium in the $\beta$-decay. Muons can be created when the chemical equilibration condition between the electron and the muon, $\mu_{e}=\mu_{\mu}$ can be fulfilled. Given the values of $\bar{\sigma}, \bar{\omega}$ and $\bar{b}_{03}$ for each $\rho$, charge neutrality of Eq. (26), chemical equilibrium of Eq. (31), $\mu_{e}=\mu_{\mu}$ and $\rho=\rho_{n}+\rho_{p}$ determine the number of particles 

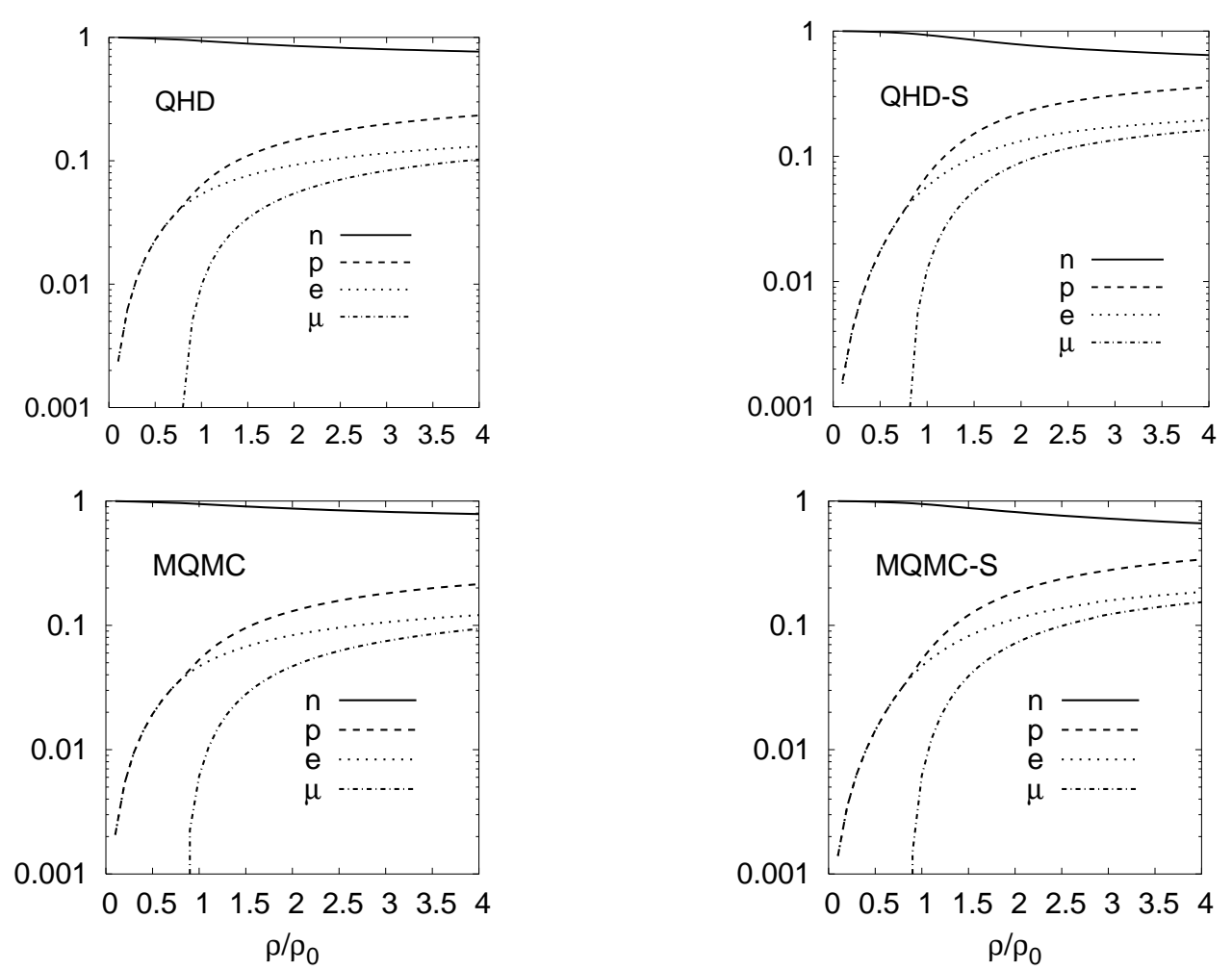

FIG. 3: The particle $(n, p, e, \mu)$ fractions in neutron stars calculated by different nuclear models are plotted against neutron star matter densities $\rho / \rho_{0}$. Since the figure for MQMC-MB is similar to that for MQMC, we do not show it here.

unambiguously. The results are shown in Fig. 3 ,

The compositions from the QHD and MQMC models are very similar to each other, but the number of the protons for the QHD-S or MQMC-S model is larger than that for the QHD or the MQMC, respectively. The ratio of the proton number to the neutron number, $\rho_{p} / \rho_{n}$ is especially important in determining the cooling mechanism of the neutron star. Two major mechanisms of the cooling are emission of the neutrinos from the interior and radiation of the photons near the surface of the neutron star. The simplest neutrino emission mechanism is the so-called direct URCA (DU) process, which is nothing but the $\beta$-decay as in Eq. (27), and it is known as the most efficient neutrino-emitting process in the interior of the neutron star. In order for this process to occur, however, the ratio $\rho_{p} / \rho_{n}$ should be larger than a critical value. The proton numbers from QHD-S and MQMC-S models increase faster than those from the QHD and MQMC models as the density increases. This implies that $\rho_{p} / \rho_{n}$ from QHD-S or MQMC-S can reach the critical value at lower densities 
and the $\beta$-decay can take place over a wider region in the interior of the neutron star than the QHD or MQMC models predict, causing a rapid cooling of the neutron star. However, a recent work on the cooling of the neutron star [18] indicates that too low a threshold density for the DU process can result in an unrealistic cooling scenario. DU process can happen when both energy and momentum conservation is satisfied in Eq. (27). Since neutrinos have energy of the thermal fluctuation around the Fermi momentum of the nucleon or electron, its momentum can be neglected. Then the momentum conservation condition for DU can be written as $k_{n}=k_{p}+k_{e}$, where $k_{i}$ being the Fermi momentum of particle " $i$ ". The threshold density $\rho_{D}$ at which DU starts to emit neutrinos is about $\rho_{D} \simeq 2.1 \rho_{0}$ for QHD and MQMC, and is about $\rho_{D} \simeq 1.6 \rho_{0}$ for QHD-S and MQMC-S. These threshold densities are too low to explain the observation of the neutron star temperature [18]. In a recent work 14], the density dependent meson masses and coupling constants are considered within the framework of relativistic mean field theory. It is shown that they obtained lower value of $\rho_{D}$ than that of our QHD model. Therefore the nuclear models we have considered in this work may need further refinement and adjustment of parameters to produce threshold densities high enough for the cooling process to be reasonable.

\section{Mass and radius of the neutron star}

The mass and the radius of the neutron star can be obtained by integrating OppenheimerVolkov equation with a given EoS. In Fig. 4 the neutron star mass with our EoS is plotted as a function of the central density $\varepsilon_{c}$ (left panel) and of the radius (right panel) in units of solar mass $M_{\odot}$. The solid line is the result from the QHD-S, the dashed line from the QHD, and the dotted line from the MQMC. QHD and MQMC give us very similar maximum mass $M_{\text {max }} \simeq 2.0 M_{\odot}$ with $R \simeq 10.8 \mathrm{~km}$ and $\varepsilon_{c} \simeq 2.5 \times 10^{15} \mathrm{~g} / \mathrm{cm}^{3}$. On the contrary, due to the stiff EoS the QHD-S gives us the maximum mass of about $2.9 M_{\odot}$ with the radius $R \simeq 13.7$ $\mathrm{km}$ and the central density $\varepsilon_{c} \simeq 1.5 \times 10^{15} \mathrm{~g} / \mathrm{cm}^{3}$. We could not extract the maximum mass of the neutron star for MQMC-S and MQMC-MB models since, as noted in the previous section, these models encounter a problem in solving the SCC at a density before reaching the maximum mass.

Recent observations of the neutron star mass from the radio pulsars give us the mass range of the neutron stars as $(0.8 \sim 2.2) M_{\odot}[19]$. The maximum mass of the QHD-S 

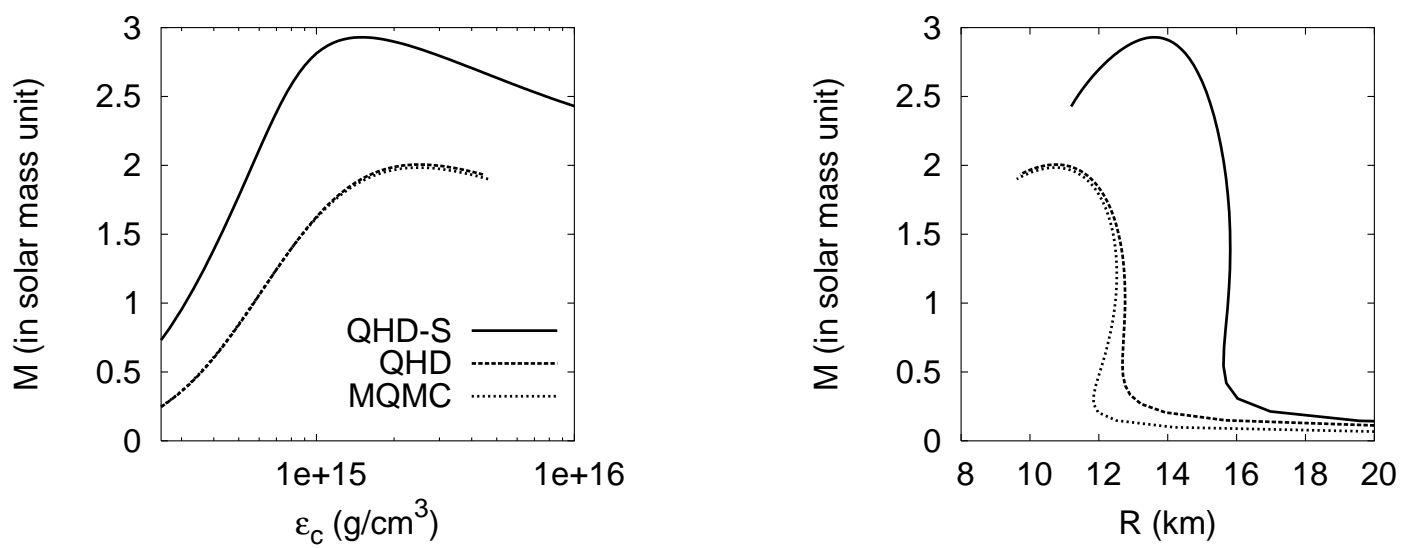

FIG. 4: Neutron star mass as a function of the central density (left) and the radius (right). The solid line is the result from the QHD-S, the dashed line from the QHD, and the dotted line from the MQMC.

is much larger than the upper limit of the observed values, so one may want to exclude the QHD-S among the models for the neutron stars. We may note, however, that more considerations are needed as follows. First, the QHD-S produces the saturation properties successfully. Secondly, we have considered only the nucleon degrees in this work, but other degrees of freedom can come in such as hyperons or the phases like meson condensation and quark matter. In general, when these exotic degrees of freedom appear, they reduce the Fermi momentum of nucleons through the $\beta$-decay of the nucleons to exotic states. This results in a smaller contribution of $P_{N}$ to the pressure, and thus the EoS becomes softer with exotic degrees of freedom than otherwise. Then the maximum mass of the neutron star becomes smaller. We have confirmed this behaviour by doing some more calculations including hyperons in the QHD and MQMC. The maximum mass of $1.7 M_{\odot}$ is obtained for $\mathrm{QHD}$, and $1.5 M_{\odot}$ for MQMC. It will be interesting to investigate how these maximum masses will change when medium effects on meson masses and coupling constants are taken into consideration. It is noted, however, that the inclusion of hyperons can give us too low a mass limit of the neutron star 20], which is incompatible with the observations. In Ref. [20], a phase transition to quark matter is considered, and the maximum mass of the neutron star is obtained to lie in the range $(1.4 \sim 1.7) M_{\odot}$. A transition to quark matter is one possibility, and at the same time transitions to other exotica are also possible. These possibilities should be considered for a better understanding. 


\section{SUMMARY}

In this work, we have investigated the properties of the neutron star matter by including the effects of meson mass changes in medium. We have explored various models at the hadron (QHD and QHD-S) and at the quark (MQMC, MQMC-S and MQMC-MB) levels. We have incorporated in-medium meson masses in two ways: one by assuming a simple function of scaling in both QHD and MQMC models, and the other by treating heavy mesons as MIT bags within the framework of MQMC model. The EoS and the particle fractions for the neutron star matter are calculated. Scaling models show appreciable difference from the models with constant meson mass or meson bag models. The EoS's from the scaling models are stiffer than those from other models. The scaling models show higher fractions of the protons than other models. Stiff EoS's from the scaling models lead to heavy and large neutron stars whose maximum mass is about $2.9 M_{\odot}$ which is larger than the observed values and the values predicted by other models by one solar mass. We may, however, note that the present work is just a first step toward the understanding of the relationship between the in-medium properties of physical quantities (mass, coupling constant and etc.) and the neutron star, and further studies need to be done.

\section{Acknowledgements}

This work is supported by Korea Research Foundation Grant (KRF-2002-042-C00014).

\section{Appendix}

In this section, we show a breakdown mechanism that occurs in solving the SCC of the QMC model. Since the SCC for MQMC-S and MQMC-MB are involved, we employ the QMC model for the purpose of a simple illustration. The SCC of the QMC model can be obtained by putting $g_{\sigma}^{B}=0$ in Eq. (17), which leads to

$$
\bar{\sigma}=3 \frac{g_{\sigma}^{q}}{m_{\sigma}^{2}} \rho_{s} C_{N}(\bar{\sigma})
$$

The breakdown takes place when $C_{N}(\bar{\sigma})$ changes the sign from positive to negative at a certain density. The numerator in the square bracket of Eq. (18) causes the sign change. 


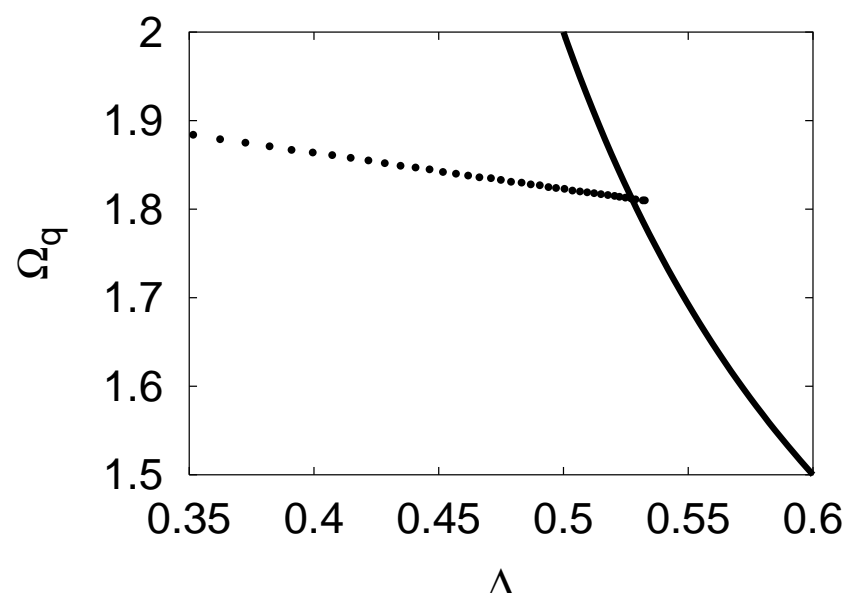

FIG. 5: The solid curve represents Eq. (39) and the dots represent $\Omega_{q}=\Omega_{q}(\Delta)$ with $R m_{q}^{*}$ obtained from the QMC calculation. In the left section divided by the solid curve, NU is greater than zero, which is allowed physically. QMC calculation has been done up to $\rho=5.3 \rho_{0}$.

Rearranging the numerator of Eq. (18), we obtain

$$
\begin{aligned}
\mathrm{NU}= & \Omega_{q}^{2}+3 \Omega_{q}\left(\Omega_{q}-1\right) R m_{q}^{*}-Z_{N}\left(\frac{1}{2} \Omega_{q}+\left(\Omega_{q}-1\right) R m_{q}^{*}\right) \\
& +\frac{1}{2}\left(R m_{q}^{*}\right)^{2}+\frac{4}{3} \pi R^{4} B\left(\frac{1}{2} \Omega_{q}+\left(\Omega_{q}-1\right) R m_{q}^{*}\right)
\end{aligned}
$$

where NU means the numerator. To show roughly how the sign of $C_{N}(\bar{\sigma})$ changes, we make the following approximations. First, all the terms in Eq. (37) can be neglected since $\left(R m_{q}^{*}\right)^{2}$ and $R^{4} B$ are very small compared to the terms in Eq. (36) in the density region we consider. Secondly, we use $Z_{N}=2$ instead of the actual value $Z_{N}=2.030$. With these approximations, we have

$$
\begin{aligned}
\mathrm{NU} & \simeq \Omega_{q}^{2}\left(3 R m_{q}^{*}+1\right)-\Omega_{q}\left(5 R m_{q}^{*}+1\right)+2 R m_{q}^{*} \\
& =\left[\left(3 R m_{q}^{*}+1\right) \Omega_{q}-2 R m_{q}^{*}\right]\left(\Omega_{q}-1\right)
\end{aligned}
$$

Since $\left(\Omega_{q}-1\right)$ is always positive, the sign change of Eq. (38) occurs at

$$
\begin{aligned}
\Omega_{q} & =\frac{2 R m_{q}^{*}}{3 R m_{q}^{*}+1} \\
& =\frac{2 \Delta}{3 \Delta-1},
\end{aligned}
$$

where $\Delta \equiv-R m_{q}^{*}$. Since $\Omega_{q}$ is always greater than 1, the solution to Eq. (39) can exist for $\Delta>1 / 3$. By taking $\Delta$ as an independent variable, we plot in Fig. $5 \Omega_{q}$ of Eq. (39) with 
the solid line. Eq. (39) represents possible points at which the sign change occurs. The values of $\Omega_{q}$ obtained from the numerical solution of Eq. (15) and the SCC of the QMC is shown in Fig. 5 by the dots. In the actual numerical calculation with the QMC model (not MQMC model), of which we have not shown anything in this paper, solutions can be obtained up to $\rho=5.3 \rho_{0}$ at which $\bar{\sigma}=34.52 \mathrm{MeV}, R=0.574$ and $\Delta=0.533$. In Fig. 5, the two curves meet at around $\Delta=0.527\left(\Omega_{q}=1.812\right)$, which corresponds to the density $\rho \simeq 5.0 \rho_{0}$. If the small terms in Eq. (37) are included, the two curves will meet at around $\rho=5.3 \rho_{0}$, which is the breakdown point of the QMC in the actual numerical calculation. Similar breakdown behaviors are observed numerically for the MQMC-S and MQMC-MB models. For this reason, we could not calculate the EoS at higher densities, and consequently could not extract the mass and the radius of the neutron stars for these models.

[1] G. E. Brown and M. Rho, Phys. Rev. Lett. 66, 2720 (1991).

[2] T. Hatsuda and S. H. Lee, Phys. Rev. C 46, R34 (1992).

[3] K. Saito, K. Tsushima and A. W. Thomas, Phys. Rev. C 55, 2637 (1997).

[4] G. Agakichiev et al., Phys. Lett. B 422, 405 (1998).

[5] K. Ozawa et al., Phys. Rev. Lett. 86, 5019 (2001).

[6] I. H. Stairs, Science 304, 547 (2004).

[7] H. Quaintrell et al., Astron. Astrophys. 401, 303 (2003).

[8] C. Song, G. E. Brown, D.-P. Min and M. Rho, Phys. Rev. C 56, 2244 (1997).

[9] K. Saito and A. W. Thomas, Phys. Rev. C 51, 2757 (1995).

[10] N. K. Glendenning, F. Weber and S. A. Moszkowski, Phys. Rev. C 45, 844 (1992).

[11] C. Fuchs, H. Lenske and H. H. Wolter, Phys. Rev. C 52, 3043 (1995).

[12] S. Typel, H. H. Wolter, Nucl. Phys. A 656, 331 (1999).

[13] B. Liu, H. Guo, M. Di Toro and V. Greco, nucl-th/0409014.

[14] E. E. Kolomeitsev and D. N. Voskresensky, nucl-th/0410063.

[15] P. A. M. Guichon, Phys. Lett. B 200, 235 (1988).

[16] X. Jin and B. K. Jennings, Phys. Rev. C 54, 1427 (1996).

[17] C. Song, D.-P. Min and M. Rho, Phys. Lett. B 424, 226 (1998).

[18] D. Blaschke, G. Grigorian and D. N. Voskresensky, A\&A 424, 979 (2004). 
[19] S. E. Thorsett and D. Chakrabarty, ApJ. 512, 288 (1999).

[20] G. F. Burgio, M. Baldo, P. K. Sahu and H.-J. Schulze, Phys. Rev. C 66, 025802 (2002). 